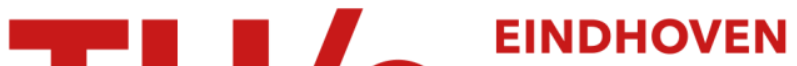 \\ UNIVERSITY OF \\ TECHNOLOGY
}

\section{Molecular dynamics study of the coverage dependence of Xe desorption from $\mathrm{Pt}(111)$}

Citation for published version (APA):

Jansen, A. P. J. (1992). Molecular dynamics study of the coverage dependence of Xe desorption from $\mathrm{Pt}(111)$. Journal of Chemical Physics, 97(7), 5205-5211. https://doi.org/10.1063/1.463819

DOI:

10.1063/1.463819

Document status and date:

Published: 01/01/1992

\section{Document Version:}

Publisher's PDF, also known as Version of Record (includes final page, issue and volume numbers)

\section{Please check the document version of this publication:}

- A submitted manuscript is the version of the article upon submission and before peer-review. There can be important differences between the submitted version and the official published version of record. People interested in the research are advised to contact the author for the final version of the publication, or visit the $\mathrm{DOI}$ to the publisher's website.

- The final author version and the galley proof are versions of the publication after peer review.

- The final published version features the final layout of the paper including the volume, issue and page numbers.

Link to publication

\section{General rights}

Copyright and moral rights for the publications made accessible in the public portal are retained by the authors and/or other copyright owners and it is a condition of accessing publications that users recognise and abide by the legal requirements associated with these rights.

- Users may download and print one copy of any publication from the public portal for the purpose of private study or research.

- You may not further distribute the material or use it for any profit-making activity or commercial gain

- You may freely distribute the URL identifying the publication in the public portal.

If the publication is distributed under the terms of Article 25fa of the Dutch Copyright Act, indicated by the "Taverne" license above, please follow below link for the End User Agreement:

www.tue.nl/taverne

Take down policy

If you believe that this document breaches copyright please contact us at:

openaccess@tue.nl

providing details and we will investigate your claim. 


\title{
Molecular dynamics study of the coverage dependence of Xe desorption from Pt(111)
}

\author{
A. P. J. Jansen \\ Laboratory for Inorganic Chemistry and Catalysis, Eindhoven University of Technology, P.O. Box 513 , \\ $5600 \mathrm{MB}$ Eindhoven. The Netherlands
}

(Received 8 April 1992; accepted 25 June 1992)

\begin{abstract}
Molecular dynamics simulations with periodic boundary conditions are used to calculate temperature-programmed desorption spectra of $\mathrm{Xe} / \mathrm{Pt}(111)$. The activation barrier is overcome using the compensating Hamiltonian method. Monte Carlo simulations are used to correct for the finite size of the simulated system. A comparison between calculated and experimental temperature-programmed desorption spectra shows that there is a strong reduction in the $\mathrm{Xe}-\mathrm{Xe}$ attraction due to substrate-mediated effects. Still, strong island formation occurs. In spite of the fact that Xe atoms in islands desorb less easily than isolated $\mathrm{Xe}$ atoms, the desorption from islands dominates at low temperature, and the desorption of isolated $\mathrm{Xe}$ atoms at high temperature. This is caused by the variation in the number of isolated $\mathrm{Xe}$ atoms and $\mathrm{Xe}$ atoms in islands as a function of temperature and coverage.
\end{abstract}

\section{INTRODUCTION}

Although the desorption of physisorbed atoms at low temperature is a relatively simple activated process, it is still only poorly understood. In a previous paper we studied the desorption of $\mathrm{Xe}$ from $\mathrm{Pd}(100)$ at low coverage. ${ }^{1}$ It was shown that good results for the desorption rate constants could be obtained with molecular dynamics (MD). The paper was however primarily on the dynamics of the desorption, as it was shown that desorption rate constants could also have been obtained from transition-state theory. We expect that this is not the case for high coverage, as it is not clear how to incorporate the adsorbate-adsorbate interactions in such a theory. Moreover, it is difficult to construct reliable potential energy surfaces when this interaction has to be included.

We refer to an excellent review paper, which recently appeared, and which discusses various theories for desorption. ${ }^{2}$ The authors point out that, because of the long time the system needs to cross the activation barries, MD is not feasible. However, a number of methods have been developed to deal with this problem. ${ }^{1,3}$ In view of the amount of detail and the absence of approximations, we think that MD is a very good method to study desorption.

We present results of an MD study on Xe desorption. Adsorbed $\mathrm{Xe}$ is interesting for its use in photoemission of adsorbed xenon (PAX) spectroscopy. ${ }^{4}$ We have investigated the effect of the Xe-Xe interaction on the desorption, and, in particular, the effect of substrate-induced changes in the $\mathrm{Xe}-\mathrm{Xe}$ potential in $\mathrm{Xe} / \mathrm{Pt}(111)$ by comparing $\mathrm{MD}$ results with temperature-programmed desorption (TPD) spectra of Siddiqui et al..$^{5}$ The $\mathrm{Xe} / \mathrm{Pt}(111)$ system has been studied before with $\mathrm{MD}$; Black and Bopp ${ }^{6}$ investigated the structure of Xe rafts, and Grimmelmann et al. ${ }^{7}$ studied the desorption at low coverage.

The method we used to calculated the desorption rate constants, and the computational details are presented in Sec. II. Section III consists of three parts; two deal with
$\mathrm{Xe}-\mathrm{Pt}$ and $\mathrm{Xe}-\mathrm{Xe}$ interactions, and one with the results of the simulations, and with a model we used to correct for the finite size of the system in the simulations. Section IV presents the conclusions.

\section{THEORY AND COMPUTATIONAL DETAILS}

The theory we used to calculate the desorption rate constant from an MD simulation has been described previously. ${ }^{1}$ We like to comment here only on how we treated the coverage dependence. The energy to remove more than one Xe atom from the $\mathrm{Pt}$ surface is approximately proportional to the number of $\mathrm{Xe}$ atoms being removed. Only little energy is gained when the Xe atoms desorb in clusters. Also entropically it is more favorable when the Xe atoms desorb individually. Hence we may assume that there is no correlation between the desorption of different $\mathrm{Xe}$ atoms, which leads to the following method, to calculate the desorption rate constant. We define a dividing surface and a compensating Hamiltonian for one particular $\mathrm{Xe}$ atom, and determine its desorption rate as in Ref. 1, treating the degrees of freedom of the other Xe atoms as those of the Pt surface. This gives us in Keck's variational reaction rate theory ${ }^{8}$ the exact relation

$$
-\frac{d P}{d t}=k P,
$$

where $P$ is the probability of the particular Xe atom being adsorbed, and $k$ is a desorption rate constant. Because of the equivalence of the $\mathrm{Xe}$ atoms we have

$$
-\frac{d \theta}{d t}=k \theta \text {, }
$$

with $\theta$ the coverage. As $k$ is coverage dependent, Eq. (2) does not mean that the desorption is a first-order process. The form of Eq. (2) is a consequence of the definition of the desorption rate constant in the theory. ${ }^{8}$ 
TABLE I. Parameters of the potentials.

\begin{tabular}{lcc}
\hline \hline $\mathrm{Pt}-\mathrm{Pt}$ & $\lambda_{\mathrm{NN}} / k_{B}$ & \\
& $\mu_{\mathrm{NN}} / k_{B}$ & $-2414 \mathrm{~K}$ \\
& $\lambda_{\mathrm{NNN}} / k_{B}$ & $3795 \mathrm{~K}$ \\
& $\mu_{\mathrm{NNN}} / k_{B}$ & $-3783 \mathrm{~K}$ \\
& $D_{e} / k_{B}$ & $362.7 \mathrm{~K}$ \\
$\mathrm{Xe}-\mathrm{Pt}$ & $R_{e}$ & $0.32 \mathrm{~nm}$ \\
& $\alpha$ & $12.27 \mathrm{~nm}$ \\
& & $158.5 \mathrm{~K}$ \\
$\mathrm{Xe}-\mathrm{Xe}$ & $D_{e} / k_{B}$ & $0.4527 \mathrm{~nm}^{-1}$ \\
& $R_{e}$ & $15.306 \mathrm{~nm}^{-1}$ \\
\hline \hline
\end{tabular}

We performed MD simulations on a box with periodic boundary conditions in two dimensions: modeling the Xecovered $\mathrm{Pt}(111)$ surface by two layers with $48 \mathrm{Pt}$ atoms each, and a number of $\mathrm{Xe}$ atoms varying from 1 to 16 (monolayer coverage). We assumed the $\sqrt{3} \times \sqrt{3} R 30^{\circ}$ structure for monolayer coverage, in which every third threefold face-centered-cubic (fcc) site is occupied, and which has a Xe-Xe nearest neighbor distance of $0.4801 \mathrm{~nm}$. We used the generalized Langevin formalism as developed by Tully to include the effect of the rest of the substrate. ${ }^{3}$ The coupling to the ghost particles was calculated by DePristo's method. ${ }^{9}$ In order to reduce the length of the simulations only the coupling of each ghost particle with one $\mathbf{P t}$ atom was retained. This coupling was scaled to get approximately the same amplitudes for the vibrations of the $\mathrm{Pt}$ atoms as when all coupling terms with the ghost particles would have been included.

The Pt-Pt interaction is given by the harmonic form

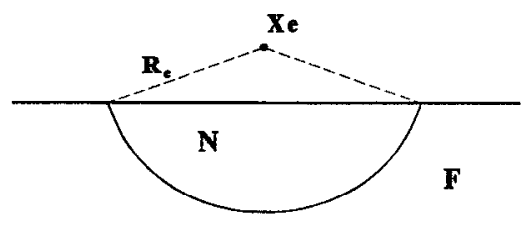

FIG. 1. Separation of the substrate into a near region $N$ and a far region $F$. The interaction of a Xe atom with the substrate consists of the lattice sum over the $\mathrm{Pt}$ atoms in $N$, and an integration over $F$, which is approximated by a continuum.

$\phi_{i j}=\frac{\lambda_{i j}}{2}\left[\left(\mathbf{u}_{i}-\mathbf{u}_{j}\right) \hat{\mathbf{r}}_{i j}\right]^{2}+\frac{\mu_{i j}}{2}\left[\left(\mathbf{u}_{i}-\mathbf{u}_{j}\right)^{2}-\left(\left(\mathbf{u}_{i}-\mathbf{u}_{j}\right) \cdot \hat{\mathrm{r}}_{i j}\right)^{2}\right]$,

where the $u$ 's are the displacements from the equilibrium positions, and $\widehat{\mathbf{r}}_{i j}$ is a unit vector connecting the equilibrium positions. The $\mathrm{Pt}$ atoms were placed at bulk positions with a nearest-neighbor distance of $0.2772 \mathrm{~nm}$. The parameters $\lambda_{i j}$ and $\mu_{i j}$ were determined for nearest and next-nearest neighbors so that they gave the correct the elastic constants and bulk specific heat (see Table I). The Xe-Pt and the Xe-Xe interactions were fitted to TPD spectra. ${ }^{10,11}$ Both interactions were represented by a Morse potential, i.e.,

$$
\phi(R)=D_{e} x(x-2), \quad \text { with } x=e^{-\alpha\left(R-R_{e}\right)} .
$$

The bond length $R_{e}$ its strength $D_{e}$, and the parameter $\alpha$ will be discussed in the next section. We used a cutoff distance $R_{c}=0.83 \mathrm{~nm}$ which was dictated by the minimal image convention. ${ }^{12}$ The interaction of a Xe atom with the $\mathrm{Pt}$ surface was calculated by summing over all $\mathrm{Pt}$ atoms within a distance $R_{c}$. We also added a contribution of the $\mathrm{Pt}$ atoms farther away as follows. We assumed that the region $F$ in Fig. 1 is a continuum with the density $\rho$ of bulk $\mathrm{Pt}$, and integrate over this region. This gives us for the contribution of $F$ to the potential

$V_{F}(z)=\left\{\begin{array}{l}\frac{\pi \rho D_{e}}{2 \alpha^{3}} e^{-\alpha\left(R_{c}-R_{e}\right)}\left[\left(2 \alpha^{2} R_{c}^{2}-2 \alpha^{2} R_{c} z+2 \alpha R_{c}-\alpha z+1\right) e^{-\alpha\left(R_{c}-R_{e}\right)}-8\left(\alpha^{2} R_{c}^{2}-\alpha^{2} R_{c} z+2 \alpha R_{c}-\alpha z+2\right)\right], \quad \text { if } \quad z \leqslant R_{c}, \\ \frac{\pi \rho D_{e}}{2 \alpha^{3}} e^{-\alpha\left(z c-R_{e}\right)}\left[(\alpha z+1) e^{-\alpha\left(z-R_{e}\right)}-8(\alpha z+2)\right], \quad \text { if } z \geqslant R_{c},\end{array}\right.$

where $z$ is the distance between the Xe atom and the surface. The usual procedure is to use the expression for $z \geqslant R_{c}$ for all $z$. This leads, however, to a double contribution from the region $N$. A similar procedure was not employed for the $\mathrm{Xe}-\mathrm{Xe}$ interaction, as it led to a discontinuous force at $R_{c}$, because of the Xe layer being two dimensional. Instead, we simply summed over all Xe-Xe pairs less than $R_{c}$ apart.

The dividing surface $S=0$ was defined by

$$
S=\frac{p_{z}^{2}}{2 m_{\mathrm{Xe}}}+V_{C}(z),
$$

where $z$ is the coordinate of the Xe atom, for which we calculated the desorption rate, and $p_{z}$ is the conjugate momentum. The potential $V_{C}$ is the potential for a Xe atom taken from a monolayer coverage. Note that, because of the cutoff in the Xe-Xe potential, this potential is at large $z$ identical to one for a bare Pt surface. Hence the desorption flux through the dividing surface is also correct for low coverages, but this particular definition yields fewer recrossings of the dividing surface. The compensating Hamiltonian is given by $H=\tau S$, with $0 \leqslant \tau<1$. The value of $\tau$ has to be chosen sufficiently small so that equilibrium can be attained before the Xe atom desorbs, and sufficiently large so that the $\mathrm{Xe}$ atom desorbs within the time the system is simulated. As has been shown before, the choice 
of $\tau$ is not very critical. ${ }^{1}$ We used $16 \mathrm{ps}$ to equilibrate the system, and then a further 200 ps for desorption.

\section{RESULTS AND DISCUSSION}

\section{A. The Xe-Pt potential}

We have performed simulations with 1 to $16 \mathrm{Xe}$ atoms, and at temperatures $T=50,100,200,500$, and $1000 \mathrm{~K}$. The advantage of this temperature range, which is much wider than the range in which desorption is found experimentally, which is between 90 and $110 \mathrm{~K}$, is that it is easier to obtain desorption rate constants at higher temperatures, so that we can obtain better statistics. The temperature dependence of the desorption rate constant could, for all coverages, be described by an Arrhenius form, even though some deviations were found which were qualitatively similar to, but much smaller than deviations reported for this system before. ${ }^{7}$ We typically did about 100 simulations at each coverage and temperature. A simulation took on average 10 minutes on a single processor of an Alliant FX/ 2816 computer. In this subsection we discuss the simulations with one $\mathrm{Xe}$ atom, which we used to obtain the $\mathrm{Xe}-\mathrm{Pt}$ potential.

A recent analysis of TPD spectra by Siddiqui et al. ${ }^{5}$ yielded activation energies that were substantially lower than the adsorption energies obtained with almost all Xe$\mathrm{Pt}$ potentials used so far. We decided to use the Xe-Pt potential of Arumainayagam et al. ${ }^{13}$ This potential was fitted to obtain good sticking probabilities, and should therefore describe the energy exchange with the surface reasonably well. However, from our simulations we found that this potential gives an adsorption energy $E_{\text {ads }} / k_{B}$ $=3115 \mathrm{~K}$, an activation energy $E_{\text {act }} / k_{B}=3117 \pm 71 \mathrm{~K}$, and a pre-exponential factor $v=10^{11.59 \pm 0.18} \mathrm{~s}^{-1}$, compared to $E_{\text {act }} / k_{B}=2883 \pm 252 \mathrm{~K}$, and $\nu=10^{12.5 \pm 0.9} \mathrm{~s}^{-1}$ from the TPD spectra. These calculated values are only just within the errors of the experiment, whereas the calculated and experimental peak maximum temperatures $T_{m}$ differ as much as $22 \mathrm{~K}$. We therefore changed $D_{e}$ and $\alpha$ of the potential to achieve the following scaling: $E_{\mathrm{ads}} \rightarrow \gamma E_{\mathrm{ads}}$ and $\ln v_{0} \rightarrow(1 / \gamma) \ln v_{0}$, where $v_{0}$ is the harmonic vibrational frequency of the Xe atom perpendicular to the surface. If we assume that $E_{\text {act }}$ is more or less proportional to $E_{\text {ads }}$, and $v$ to $v_{0}$, then this scaling for $\gamma<1$ reduces $E_{\text {act }}$ and increases $v$, which has the desired effect. Changing only $E_{\text {ads }}$ or $v_{0}$, to obtain a reasonable $T_{m}$ in the TPD spectrum, yielded $E_{\text {act }}$ well below or $v$ much above the experimental value of Siddiqui et al. ${ }^{5}$ The final Xe-Pt potential parameters are given in Table I. This potential gives $E_{\mathrm{act}} / k_{B}$ $=2851 \pm 59 \mathrm{~K}$ and $v=10^{11.99 \pm 0.27} \mathrm{~s}^{-1}$. The errors in the calculated activation energy and the pre-exponential factor are three times the standard deviation from the linear regression fit with an Arrhenius form to the simulation results.

\section{B. The Xe-Xe potential}

Increasing the coverage up to monolayer shifts $T_{m}$ to higher temperatures by about $10 \mathrm{~K}$. This shift is caused by the Xe-Xe interactions. For the direct interaction between
$\mathrm{Xe}$ atoms a good potential is known derived by Barker $e t$ al. ${ }^{14}$ Using this potential and assuming a uniform heating rate of $1 \mathrm{~K} \mathrm{~s}^{-1}$ we found a shift of about $30 \mathrm{~K}$. Even though experimentally a somewhat different heating rate was used, the difference in shifts clearly indicates that the $\mathrm{Xe}-\mathrm{Xe}$ potential has to be substantially modified. Work by McLachlan ${ }^{15}$ and by Kohn and Lau ${ }^{16}$ shows that the dispersion interaction with a substrate modeled by a continuum may be reduced by as much as $1 / 3$ by substratemediated effects. On the other hand Girard and Girardet ${ }^{17}$ found an increase for the dispersion interaction when a discrete model for the surface was used. This model, however, seems not appropriate for the $\mathrm{Pt}(111)$ as it would only increase the shift. If we take only $2 / 3$ of the dispersion interaction, then the shift decreases to about $20 \mathrm{~K}$.

An additional repulsion between the Xe atoms may be present because of charge transfer. ${ }^{18}$ This charge may be estimated from the change in the work function. Using a change in the work function of $0.6 \mathrm{~V}$ at full coverage, ${ }^{19}$ and a charge separation of $0.245 \mathrm{~nm}$, which is the Xe-surface distance, we find $q_{\mathrm{Xe}}=0.022 e$. This gives an additional Coulomb repulsion which decreases the shift in $T_{m}$ to about $13 \mathrm{~K}$. We fitted this potential with a Morse form (see Table I), and used it in all subsequent simulations. Even though the physical ground on which this potential is based is suspect, we feel that the magnitude of the interaction between Xe atoms on $\mathrm{Pt}(111)$ is reasonably well represented.

\section{The desorption parameters}

For all coverages we could fit the desorption rate constant $k$ of Eq. (2) by an Arrhenius form

$$
k=v e^{-E_{\text {act }} / k_{B} T} \text {. }
$$

The values for the activation energy and the preexponential factor as a function of the number of Xe atoms are shown in Fig. 2. We see that the activation energy increases with increasing coverage. This is cxpected as the $\mathrm{Xe}$ atoms attract each other. Also the pre-exponential factor increases somewhat, indicating a small compensating effect. This may be due to the increased frequency of the vibration perpendicular to the surface, or to the increase in the number of collisions between the Xe atoms. From Fig. 2 it is in principle possible to calculate the TPD spectra. The result is shown in Fig. 3. We assumed a cubic dependence of the activation energy, and a linear dependence of the pre-exponential factor as shown by the dashed lines in Fig. 2. The spectra suggest a zero-order process, except for high coverages where the desorption starts only very late. This is caused by the sharp increase of the activation energy at high coverage.

The TPD spectra of Fig. 3 are, however, probably not correct for the following reason. At the temperatures where desorption is found in the TPD experiment the simulations show island formation. Figure 2 indicates that desorption for Xe atoms in an island is slower than for an isolated $\mathrm{Xe}$ atom, as the increase of the activation energy dominates the increase of the pre-exponential factor. Whether the desorption is mainly from islands or of iso- 

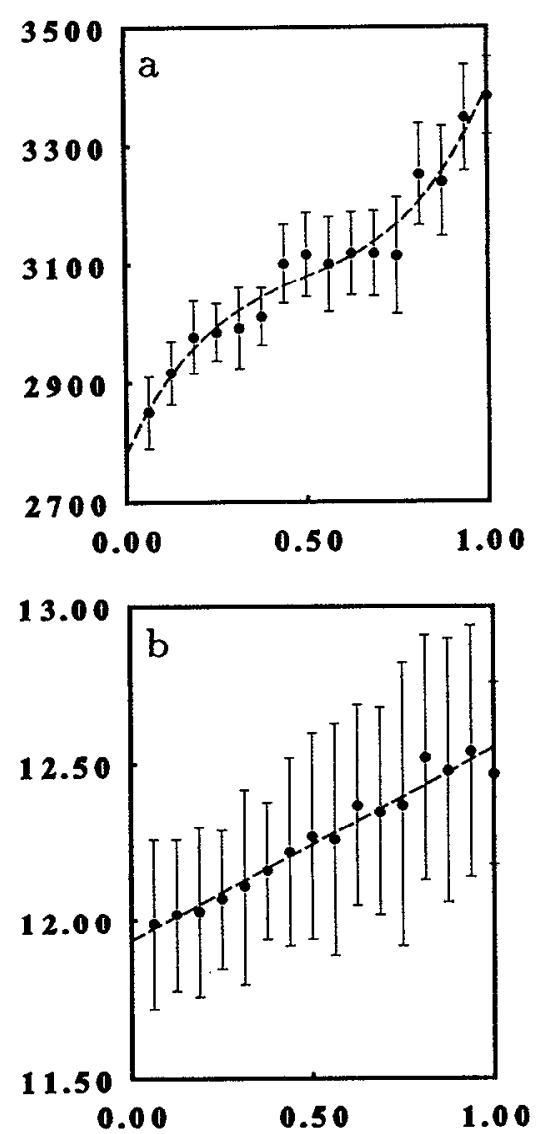

FIG. 2. (a) The activation energy $E_{\mathrm{act}} / k_{B}$ (in $\mathrm{K}$ ) and (b) the preexponential factor $\log _{10} v$ (in $\mathrm{s}^{-1}$ ) as a function of the number of $\mathrm{Xe}$ atoms, scaled to monolayer coverage, as obtained directly from the simulations. The error bars depict three times the standard deviation of a linear regression fit with an Arrhenius form. The dashed lines are fits (see text).

lated atoms depends at a certain coverage on the relative number of isolated Xe atoms to the number of Xe atoms in islands. As the size of the system used in the simulation is small compared to a real surface, a Xe atom has less room to be isolated. Thus we have to correct for finite size effects.

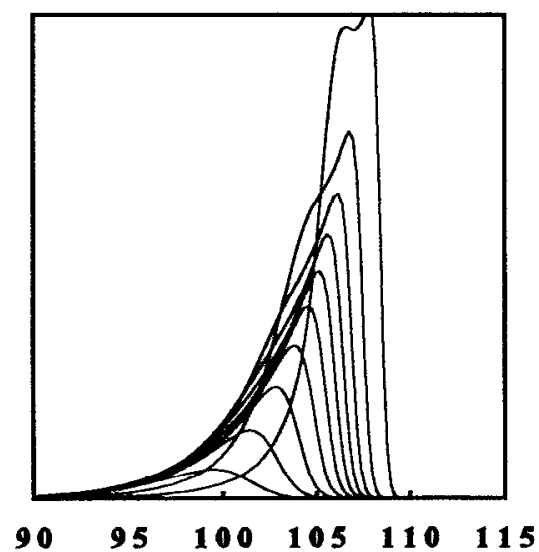

FIG. 3. Calculated TPD spectra before finite-size correction. Shown are spectra for initial coverage from 0.1 to $1.0 \mathrm{in}$ steps of 0.1 . The temperature increase is $1 \mathrm{~K} \mathrm{~s}^{-1}$.
TABLE II. The activation energy and the pre-exponential factor in the Arrhenius form with which $\vec{k}(n)$ is fitted. The errors are three times the standard deviation.

\begin{tabular}{lll}
\hline \hline$n$ & $E_{\text {act }} / k_{B}$ & $v$ \\
\hline 0 & $2851 \pm 59 \mathrm{~K}$ & $10^{11.99 \pm 0.27} \mathrm{~s}^{-1}$ \\
1 & $2910 \pm 51 \mathrm{~K}$ & $10^{11.94 \pm 0.23} \mathrm{~s}^{-1}$ \\
2 & $2979 \pm 44 \mathrm{~K}$ & $10^{12.08+0.20} \mathrm{~s}^{-1}$ \\
3 & $3039 \pm 56 \mathrm{~K}$ & $10^{12.12 \pm 0.25} \mathrm{~s}^{-1}$ \\
4 & $3153 \pm 107 \mathrm{~K}$ & $10^{12.41 \pm 0.48} \mathrm{~s}^{-1}$ \\
5 & $3321 \pm 128 \mathrm{~K}$ & $10^{12.63 \pm 0.58} \mathrm{~s}^{-1}$ \\
6 & $3383 \pm 64 \mathrm{~K}$ & $10^{12.46 \pm 0.29} \mathrm{~s}^{-1}$ \\
\hline
\end{tabular}

We have assumed that the following Ising model is valid. ${ }^{20}$ The preferred site for the Xe atom is the threefold fcc site. The Xe atoms are statistically distributed over these sites. The energy of a certain configuration of the $\mathrm{Xe}$ atoms is given by

$$
E_{\mathrm{conf}}=N_{N N} \phi_{\mathrm{Xe}-\mathrm{Xe}}\left(R_{\mathrm{NN}}\right),
$$

where $N_{\mathrm{NN}}$ is the number of nearest neighbors in the configuration and $R_{\mathrm{NN}}$ is the nearest neighbor distance. The cutoff distance of the Xe-Xe potential is such that we have only nearest-neighbor interaction. The probability of a configuration is simply given by a Boltzmann factor. The desorption rate for a particular $\mathrm{Xe}$ atom depends on the number of nearest neighbors it has. We can thus write the desorption rate constant that we obtained from the simulation as follows:

$$
k_{\mathrm{sim}}(\theta)=\sum_{n=0}^{6} P_{\mathrm{sim}}(n, \theta) \bar{k}(n),
$$

where $\bar{k}(n)$ is the desorption rate constant for a Xe atom with $n$ ncarcst neighbors, and $P_{\text {sim }}(n, \theta)$ is the probability for $n$ nearest neighbors at coverage $\theta$ for a system with the periodic boundary conditions of the simulation. Note that $\bar{k}(n)$ does not depend on the size of the system. As the box of the simulation is relatively small, we can calculate $P_{\text {sim }}(n, \theta)$ exactly. With the values for $k_{\text {sim }}(\theta)$ of the simulation we can then determine $\bar{k}(n)$. As we have done simulations at more coverages than there are $\bar{k}(n)$ 's, we use a least-squares fit procedure for $\ln k_{\mathrm{sim}}$. We found for each temperature a very good fit. This means that our model correctly describes the desorption as simulated. Again, we could use an Arrhenius form to describe the temperature dependence of the $\vec{k}(n)$ 's. The results are shown in Table II.

The desorption rate constants for a real system $k(\theta)$ can be obtained from

$$
k(\theta)=\sum_{n=0}^{6} P(n, \theta) \bar{k}(n),
$$

where $P(n, \theta)$ is the probability for $n$ nearest neighbors at a real surface. We have obtained these probabilities from a Monte Carlo simulation for systems with $N_{S}=16,64,256$, and 1024, where $N_{S}$ is the number of sites in the Monte 

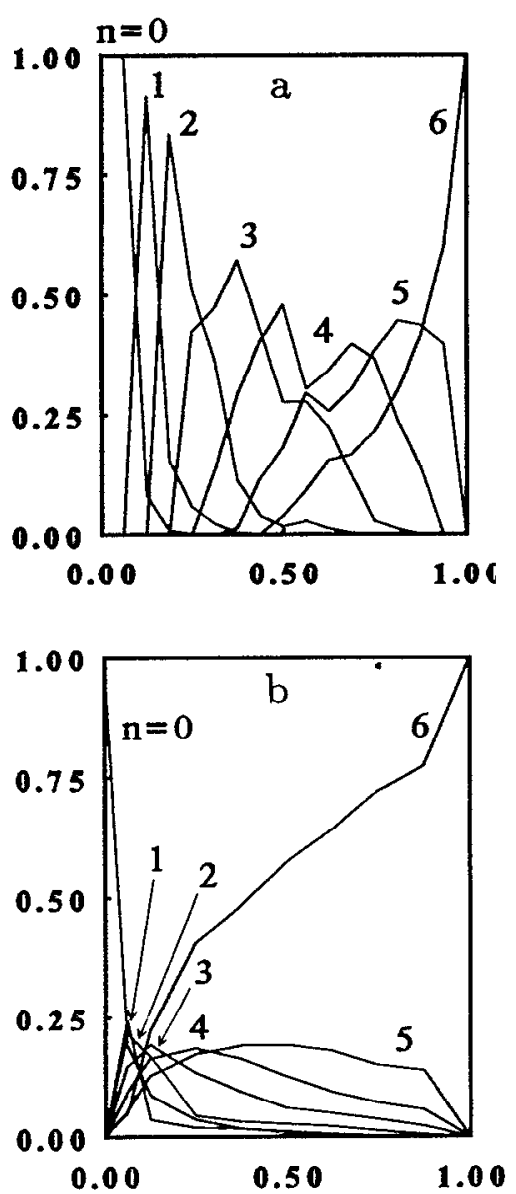

FIG. 4. The probabilities (a) $P_{\text {sim }}$ and (b) $P$ for different nearest neighbors as a function of coverage at $T=100 \mathrm{~K}$.

Carlo simulation, and linear extrapolation for $1 / N_{S} \rightarrow 0$. The values of $P_{\text {sim }}$ and $P$ are very different as can be seen in Fig. 4.

With the procedure above we can calculate the TPD spectra (see Fig. 5). A comparison with experimental spectra show that $T_{m}$ is somewhat too high. There seems to be

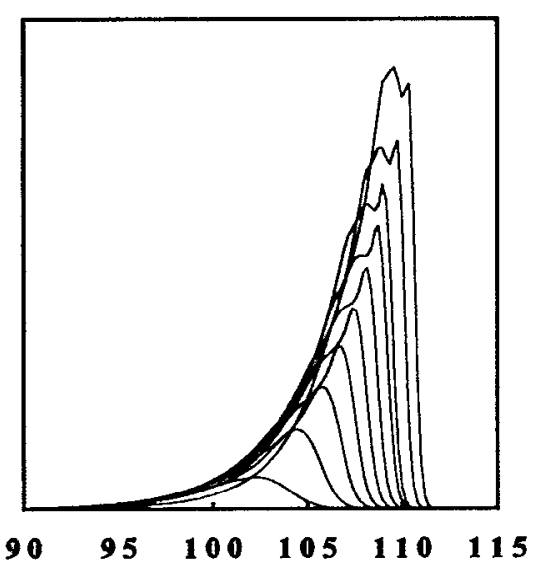

FIG. 5. Calculated TPD spectra after finite-size correction. Shown are spectra for initial coverage from 0.1 to 1.0 in steps of 0.1 . The temperature increase is $1 \mathrm{~K} \mathrm{~s}^{-1}$.

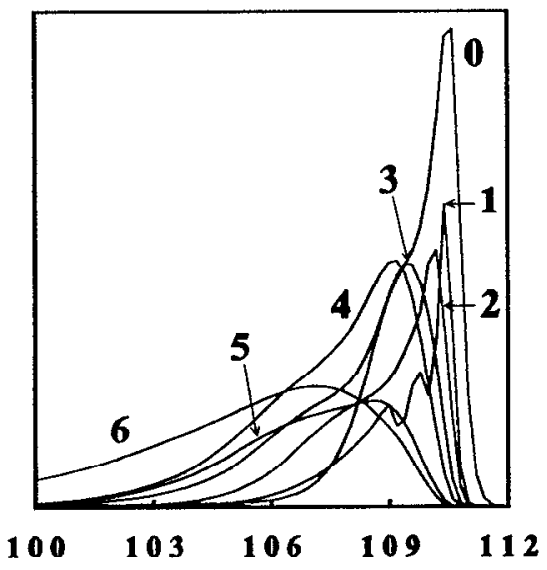

FIG. 6. Contributions of $\mathrm{Xe}$ atoms with different number of nearest neighbors to the calculated TPD spectrum after finite-size correction with initial monolayer coverage. The temperature increase is $1 \mathrm{~K} \mathrm{~s}^{-1}$.

two reasons for this. First, we have assumed a constant temperature increase of $1 \mathrm{~K} \mathrm{~s}^{-1}$, whereas experimentally a nonlinear temperature ramp was used. The peak maximum temperatures are very sensitive to the shape of this ramp, as we are in a temperature range at the beginning of the ramp (see Fig. 1 of Ref. 5). An attempt to simulate the ramp at low coverage failed because all Xe desorbed before the temperature could increase by any appreciable amount. Second, we still overestimate the $\mathrm{Xe}-\mathrm{Xe}$ interaction. Even at the lowest coverage that was used experimentally $(\theta$ $=0.05$ ), we find islanding. As we used this coverage to get the $\mathrm{Xe}-\mathrm{Pt}$ potential, assuming no $\mathrm{Xe}-\mathrm{Xe}$ interactions, we find that the peaks shift to higher temperatures. The peaks are also shifted to somewhat higher temperatures as in Fig. 3 . This is due to the fact that the Xe atoms have more neighbors on average on a real surface than in the simulations (cf. Fig. 4).

A second difference between the calculated and the experimental spectra is that in the calculated spectra there may be a shoulder or more than one peak. This can be shown to be caused by desorption of Xe atoms with different numbers of nearest neighbors. Similar features can be seen in Fig. 3. Figure 6 shows the contribution of Xe atoms with different number of nearest neighbors to a TPD spectrum. We see that Xe atoms with fewer nearest neighbors contribute most at higher temperatures. This was found at all coverages. This seems surprising as the Xe atoms with fewer nearest neighbors have a lower activation energy. What is happening is that desorption from islands dominates, because there are too few isolated $\mathrm{Xe}$ atoms. But then as the coverage decreases the number of isolated $\mathrm{Xe}$ atoms suddenly increases. If the initial coverage is low, then most $\mathrm{Xe}$ atoms desorb when they have few nearest neighbors. Their contribution decreases however with increasing initial coverage.

Both the calculated and the experimental TPD spectra depend critically on the Xe-Xe interaction. A weaker interaction than given in Table I would give a smaller shift of $T_{m}$, but might result in first-order TPD spectra. A stronger 
interaction gives the larger shifts mentioned before. The parameters of the interaction we used in the simulations seem to be just in the small range which gives small shifts and zero-order TPD spectra in agreement with experiment.

The desorption rate constant is generally written as

$$
-\frac{d \theta}{d t}=v \theta^{n} e^{-E_{\mathrm{act}} / k_{B} T},
$$

where $n$ is the order of the reaction. We have remarked that there is no inconsistency with Eq. (2) as the desorption rate constant is coverage dependent. We can write our desorption rate in this form if we use the following definition:

$$
n \equiv \frac{\partial \ln (k \theta)}{\partial \ln \theta}
$$

for the order,

$$
E_{\mathrm{act}} \equiv-\frac{\partial \ln k}{\partial\left(1 / k_{B} T\right)}
$$

for the activation energy, and

$$
v \equiv k e^{E_{\text {act }} / k_{B} T} \theta^{1-n}
$$

for the pre-exponential factor. Note that all these quantities become temperature and coverage dependent. The calculated TPD spectra suggest a zero-order process. However, using Eq. (11) we find remarkable changes in the order during the desorption process. Only the right part of the desorption peaks are caused for all initial coverages by the same process; a first-order process due to desorption of isolated Xe atoms. This is to be expected; the process must turn into a first-order process when the coverage becomes low.

The activation energy varies smoothly during the TPD experiment. For all initial coverages large than 0.1 the activation energy is, up to about the peak maximum temperature, between 3200 and $3400 \mathrm{~K}$. At about the peak maximum temperature it suddenly decreases to $2847 \mathrm{~K}$, i.e., the activation energy for desorption of an isolated Xe atom. The order behaves very irregular, however. At initial coverages of about 0.1 and larger than 0.4 we found that the order is negative at low temperature. This indicates a strong suppression of the desorption due to $\mathrm{Xe}-\mathrm{Xe}$ attraction, which results in an increased island formation at increased coverage. Then, as the desorption rate increases, the order oscillates. After the peak maximum temperature has been reached the order suddenly becomes one. The pre-exponential factor shows similar behavior, because it is coupled to the order via Eq. (14), and because the desorption rate constant and the activation energy behave smoothly. The variation of the activation energy, the order, and the pre-exponential factor are shown in Fig. 7. It seems that, because of the erratic behavior of these quantities, expression (11) has little meaning for the Xe desorption from $\operatorname{Pt}(111)$.
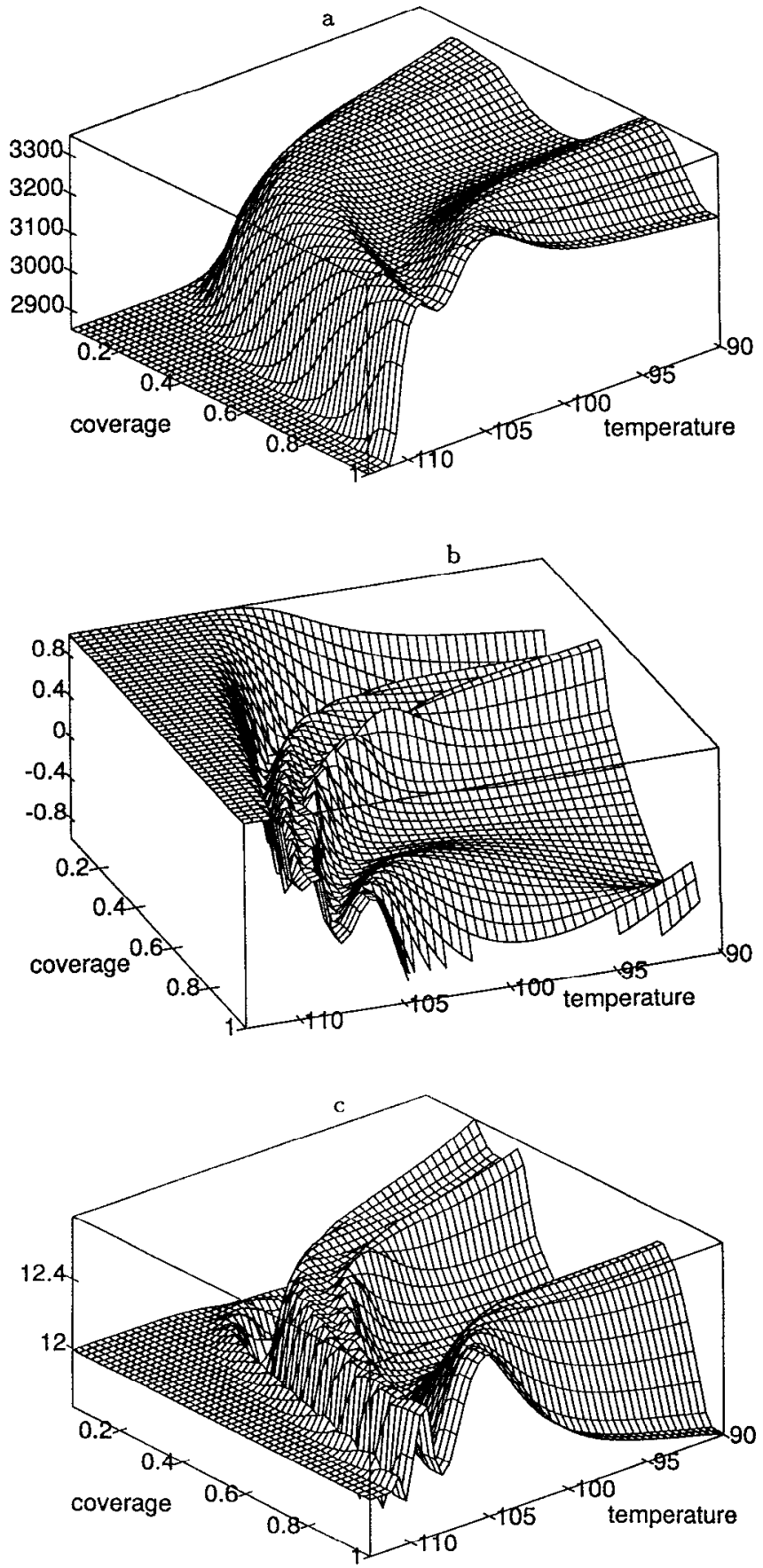

FIG. 7. Variation of (a) the activation energy $E_{\mathrm{act}} / k_{B}$ (in $\mathrm{K}$ ), (b) the order $n$, and (c) the pre-exponential factor $\log _{10} v\left(\right.$ in $s^{-1}$ ) in the TPD experiment as a function of temperature and initial coverage. The temperature increase is $1 \mathrm{~K} \mathrm{~s}^{-1}$. The flat part at high temperature is due to the first-order process that results when the coverage has become very low.

\section{CONCLUSIONS}

We calculated TPD spectra for $\mathrm{Xe} / \mathrm{Pt}(111)$. Molecular dynamics with periodic boundary conditions was used to simulated desorption for various coverages. We corrected for the finite size of the box in the simulations with an Ising model which was solved using Monte Carlo. The desorption rate was decomposed into contributions from $\mathrm{Xe}$ atoms with different numbers of nearest neighbors. 
There seems to be a large difference between the direct and the effective interaction for $\mathrm{Xe}$ atoms adsorbed on $\mathrm{Pt}(111)$. The shift in the peak maximum temperature that is found experimentally is only one-third of the shift that is found when no corrections are made for substratemediated $\mathrm{Xe}-\mathrm{Xe}$ interaction. A reduction of the dispersion interaction and a charge transfer for the substrate that leads to an additional Coulomb repulsion between Xe atoms yield the correct shift. Even with this reduced Xe-Xe interaction strong island formation takes place.

The calculated TPD spectra suggest a zero-order process in accord with experiment, but analysis shows that the order fluctuates. The same holds for the pre-exponential factor. The activation energy varies more smoothly. The fluctuations are caused by the variation in the probabilities that $\mathrm{Xe}$ atoms have a specific number of nearest neighbors as a function of coverage and temperature. These probabilities are such that $\mathrm{Xe}$ atoms with few nearest neighbors contribute at high temperatures and those with many nearest neighbors contribute at low temperatures, even though the activation energy for desorption of the former is lower than that of the latter. This is different from the usual picture of a zero-order process where the rate determining step is diffusion of an atom out of an island, and only isolated $\mathrm{Xe}$ atoms desorb.

There are two weak points in the present study. The first one is the Ising model. As it can fit the simulation results, it seems an appropriate model. However, some results, like the shoulders and the extra peaks in the calculated TPD spectra, seem uncomfortably sensitive to the precise values of the probabilities for the number of nearest neighbors. The second weak point is the Xe-Xe potential.
It is the only quantity in the Ising model that determines these probabilities. The shift in the peak maximum temperature indicates that substrate-mediated interactions are very important. Unfortunately, no good theory of the interaction, which is indispensable for understanding coverage-dependent effects of TPD spectra, exists.

\footnotetext{
${ }^{1}$ A. P. J. Jansen, J. Chem. Phys. 94, 8444 (1991).

${ }^{2}$ S. J. Lombardo and A. T. Bell, Surf. Sci. Rep. 13, 1 (1991).

${ }^{3}$ J. C. Tully, J. Chem. Phys. 73, 1975 (1980).

${ }^{4} \mathrm{~K}$. Wandelt, in Thin Metal Films and Gas Adsorption (Studies in Surface Science and Catalysis), edited by P. Wissmann (Elsevier, Amsterdam, 1987), Vol. 32.

${ }^{5}$ H. R. Siddiqui, P. J. Chen, X. Guo, and J. T. Yates, Jr., J. Chem. Phys. 92, 7690 (1990).

${ }^{6}$ J. E. Black and P. Bopp, Surf. Sci. 182, 98 (1987).

${ }^{7}$ E. K. Grimmelmann, J. C. Tully, and E. Helfand, J. Chem. Phys. 74, 5300 (1981).

${ }^{8}$ J. C. Keck, Adv. Chem. Phys. 13, 85 (1967).

${ }^{9}$ A. E. DePristo, Surf. Sci. 141, 40 (1984).

${ }^{10}$ Landolt-Börnstein, NS III/18 (Springer, Berlin, 1984).

${ }^{11} \mathrm{~J}$. de Launay, in Solid State Physics, edited by F. Seitz and D. Turnbull (Academic, New York, 1956), Vol. 2.

${ }^{12}$ M. P. Allen and D. J. Tildesley, Computer Simulation of Liquids (Clarendon, Oxford, 1987).

${ }^{13}$ C. R. Arumainayagam, R. J. Madix, M. C. McMaster, V. M. Suzawa, and J. C. Tully, Surf. Sci. 226, 180 (1990).

${ }^{14}$ J. A. Barker, R. O. Watts, J. K. Lee, T. P. Schafer, and Y. T. Lee, J. Chem. Phys. 61, 3081 (1974).

${ }^{15}$ A. D. McLachian, Mol. Phys. 7, 381 (1964).

${ }^{16}$ W. Kohn and K. H. Lau, Solid State Commun. 18, 553 (1976).

${ }^{17}$ C. Girard and C. Girardet, Surf. Sci. 195, 173 (1988).

${ }^{18}$ B. E. Nieuwenhuys and W. M. H. Sachtler, J. Colloid Interface Sci. 58, 65 (1977).

${ }^{19}$ S.-I. Ishi, Thin Solid Films 201, 373 (1991).

${ }^{20}$ Phase Transitions and Critical Phenomena, edited by C. Domb and M. S. Green (Academic, London, 1976).
} 